\title{
Case Report: Infantile Fibrosarcoma Histologically Mimicking a Benign Soft-Tissue Tumor
}

\author{
Sam Hajialiloo Sami ${ }^{1}$ (D), Farshad Zandrahimi ${ }^{*}$ (D), Mohamad Reza Heidarikhoo' ${ }^{\text {(D) }}$, Mahsa Zahmatkesh ${ }^{1}$ (D), Mansour Karimi ${ }^{1}$ (D, Hossein \\ Gholampour ${ }^{1}$
}

1. Bone and Joint Reconstruction Research Center, Shafa Yahyaian Hospital, Iran University of Medical Sciences, Tehran, Iran

\begin{tabular}{|c|c|}
\hline $\begin{array}{l}\text { Use your device to scan } \\
\text { and read the article online }\end{array}$ & \\
\hline 口pitis & $\begin{array}{l}\text { coma Histologically Mimicking a Benign Soft-Tissue Tumor. Journal of Research in Orthopedic Science. 2020; 7(4):179-184. } \\
\text { http://dx.doi.org/10.32598/JROSJ.7.4.726.1 }\end{array}$ \\
\hline 口及4 & doi: : http://dx.doi.org/10.32598/JROSJ.7.4.726.1 \\
\hline
\end{tabular}

\section{(c) (1) (3)}

Article info:

Received: 16 Aug 2020

Revised: 24 Aug 2020

Accepted: 05 Oct 2020

Available Online: 01 Nov 2020

Keywords:

Infantile fibrosarcoma, Soft-Tissue tumor,

Hemangioma

\begin{abstract}
A B S T R A C T
Infantile fibrosarcoma is a rare soft-tissue neoplasm, which may render a diagnostic challenge leading to misdiagnosis and consequently an inappropriate treatment of patients. This study reports a case of infantile fibrosarcoma that mimicked a hemangioma in an 11-month-old girl. As the lesion signal in the MRI was not consistent with the diagnosis of hemangiomas, we performed a core needle biopsy, which its result was consistent with the diagnosis of infantile fibrosarcoma. The lesion was initially treated with surgical resection. However, the lesion recurred one year after the surgery. The recurrence was managed with debulking surgery. The fifth finger was necrotized during the hospitalization after the relapse surgery. Finally, the necrotic finger was amputated. Also, adjuvant chemotherapy was used to prevent further relapses. The 1-year follow-up of the patient was recurrence-free. These findings highlight the importance of considering infantile fibrosarcoma when an infant presents with a lesion that clinically mimics a vascular lesion.
\end{abstract}

\section{Introduction}

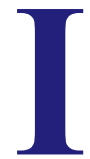

nfantile fibrosarcoma is a rare soft-tissue malignancy that presents either at birth or during the first five years of life [1]. The incidence of infantile fibrosarcoma is reported as five cases per million infants [2]. It is a spindle cell tumor similar to the adult-type but with a better prognosis [3]. Surgical excision is considered the mainstay of treatment. Also, adjuvant therapy is generally kept for patients with positive surgical margins [4].
The diagnosis of infantile fibrosarcoma could be complicated owing to its rare incidence and clinical and histological similarity to benign soft-tissue tumors, such as hemangiomas. Consequently, studies have frequently reported the misdiagnosis of infantile fibrosarcoma with benign soft-tissue tumors [5]. Further awareness is required to avoid the undertreatment of patients with infantile fibrosarcoma that might be misdiagnosed as benign soft-tissue tumors.

In this study, we report a case of infantile fibrosarcoma that clinically mimicked a hemangioma lesion in an 11-month-old infant girl.

\section{* Corresponding Author:}

Farshad Zandrahimi, PhD.

Address: Bone and Joint Reconstruction Research Center, Shafa Yahyaian Hospital, Iran University of Medical Sciences, Tehran, Iran

Phone: +98 (21) 33542000-8

E-mail:farshad_z1984@yahoo.com 

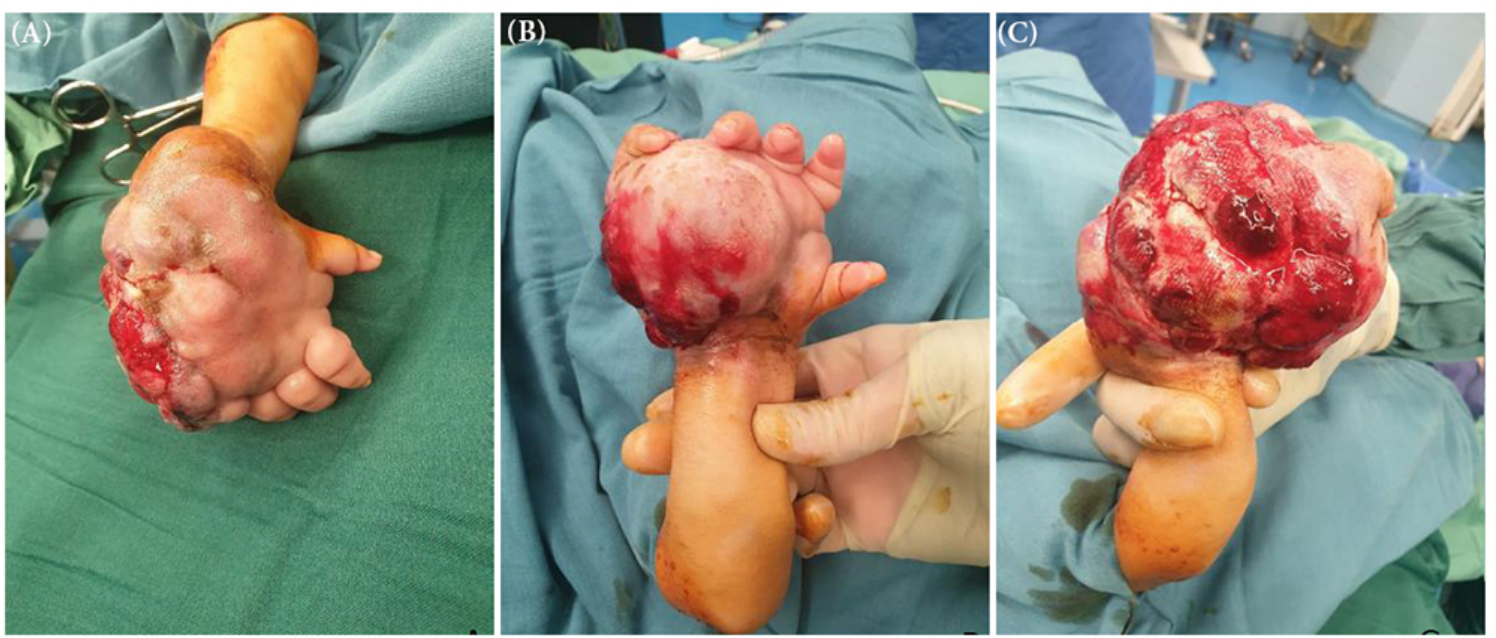

Orthopedic Science

Figure 1. Preoperative photography showing a mass in the palmar side of the right hand of an 11-month-old infant girl

A: Dorsal side; B: Volar side; C: Ulnar side

\section{Case Presentation}

An 11-month-old infant girl presented with a mass at the palmar side of her right hand since birth (Figure 1). The mass size was $7 \times 7 \mathrm{~cm}$ at presentation, however, it was growing gradually. On physical examination, the mass was firm, immobile, and palpable, with discrete borders and mild tenderness. Besides, the movement of the patient's fingers was limited. The laboratory tests were in the normal ranges; no lymphadenopathy was detectable. Based on this information, the diagnosis of the hemangioma was suspected. However, the lesion was more firm than expected.

Also, in the MRI, the signal was more heterogeneous than what was seen in hemangiomas. Therefore, a core needle biopsy was ordered for the patient. The histologic examination of the biopsy sample was consistent with the diagnosis of infantile fibrosarcoma. Subsequently, the lesion was surgically resected, and the extracted mass was sent for histologic assessment, which confirmed the diagnosis of infantile fibrosarcoma (Figure 2).

The mass recurred one year after the index surgery. At this time, the serum levels of alkaline phosphatase (ALP) and lactic dehydrogenase (LDH) were elevated to $454 \mathrm{U} / \mathrm{L}$ and $490 \mathrm{U} / \mathrm{L}$, respectively (ALP normal range, 44-147 U/L; LDH normal range, 140-280 U/L). Other laboratory indices, such as white blood cell (WBC), Erythrocyte Sedimentation Rate (ESR), C-Reactive Protein (CRP), serum glutamic-oxaloacetic transaminase (SGOT), serum glutamic pyruvic transaminase (SGPT), calcium, phosphorous, and platelet count were within the normal ranges.

Considering the young age of the patient, a debulking surgery was performed instead of the hand amputation to avoid lifetime disability (Figure 3). The histologic anal-
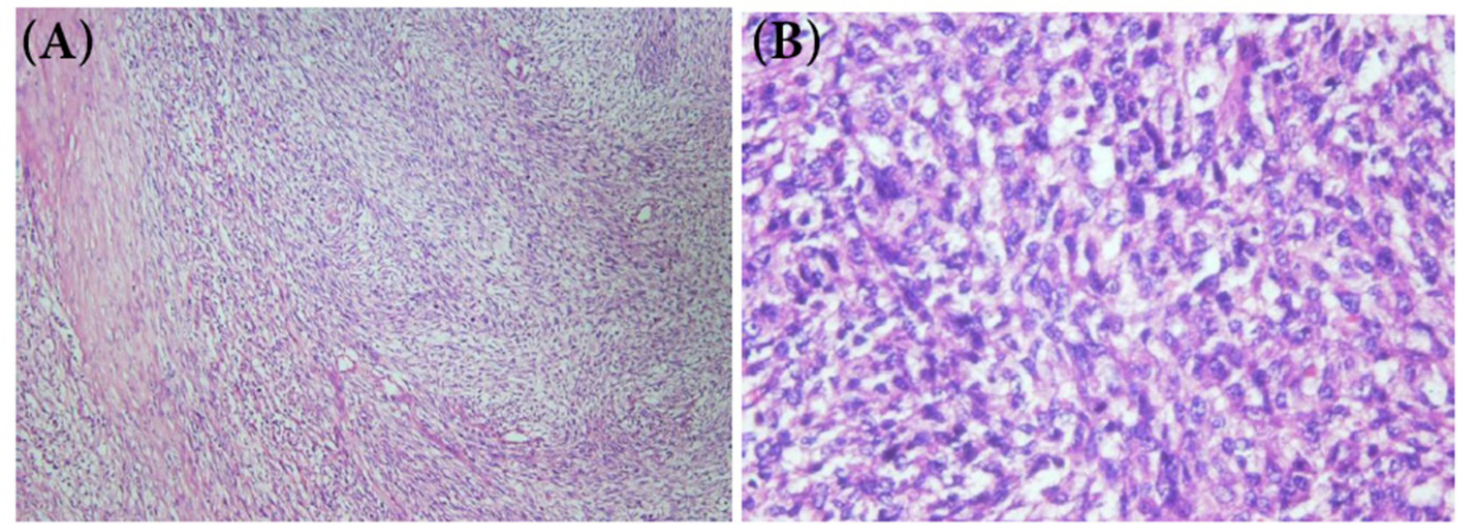

Figure 2. Histologic Examination of the extracted tissue, consistent with the diagnosis of fibrosarcoma

Orthopedic Science

A: $\times 10$ magnification; $\mathrm{B}: \times 40$ magnification 


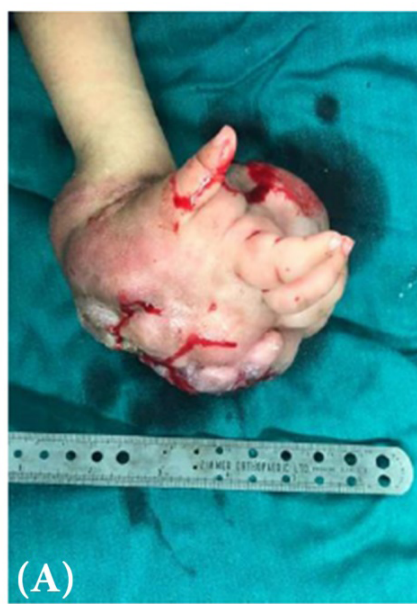

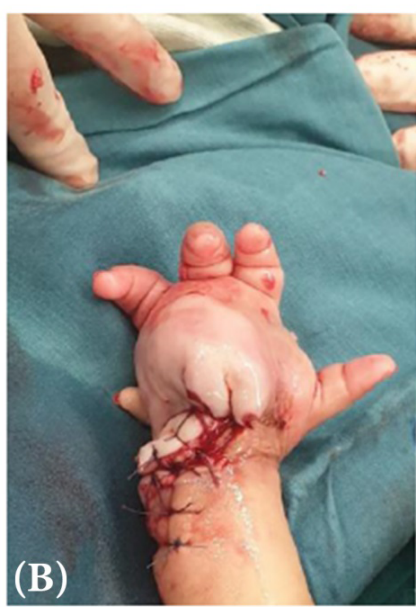

(B)

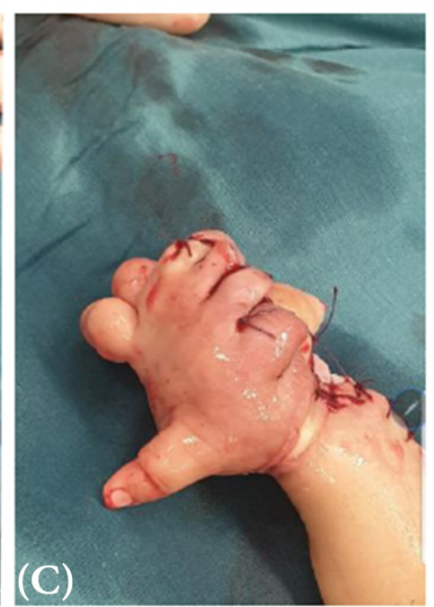

Orthopedic Science

Figure 3. Recurrence of the lesion and debulking surgery result

A: The recurrence of the lesion one year after the index surgery; B: Volar side post-debulking photography; C: Dorsal side post-debulking photography

ysis was consistent with the diagnosis of fibrosarcoma. Therefore, the metastatic workup was done, which was negative. Adjuvant chemotherapy was used to prevent further recurrence.

During the hospitalization after the second surgery, the fifth finger of the involved hand became necrotic and was amputated (Figure 4). However, the patient's hand was saved. The 1-year follow-up of the patient was recurrence-free (Figure 5).

\section{Discussion}

In this report, we presented a case of infantile fibrosarcoma that clinically mimicked a hemangioma, in an 11-month-old girl. However, the histopathologic examination was consistent with the diagnosis of infantile fibrosarcoma. The lesion was managed with surgical re- section. One year after the index operation, the tumor recurred and was managed with debulking surgery. Also, the fifth finger was necrotized during the hospitalization after the second surgery. The necrotic finger was amputated. Moreover, adjuvant chemotherapy was used to prevent further relapses. The 1-year follow-up of the patient was recurrence-free.

Several studies have reported the misdiagnosis of infantile fibrosarcoma with other tumors. Enos et al. described a neoplastic mass involving the left distal forearm of an infant boy at his seven weeks of life. The lesion was a multinodular lesion and continuously enlarging. According to ultrasound studies, the initial diagnosis is presumed to be a vascular lesion. However, the skin biopsy revealed the diagnosis of fibrosarcoma. This case highlighted the unusual cutaneous presentation of congenital infantile fibrosarcoma [6].

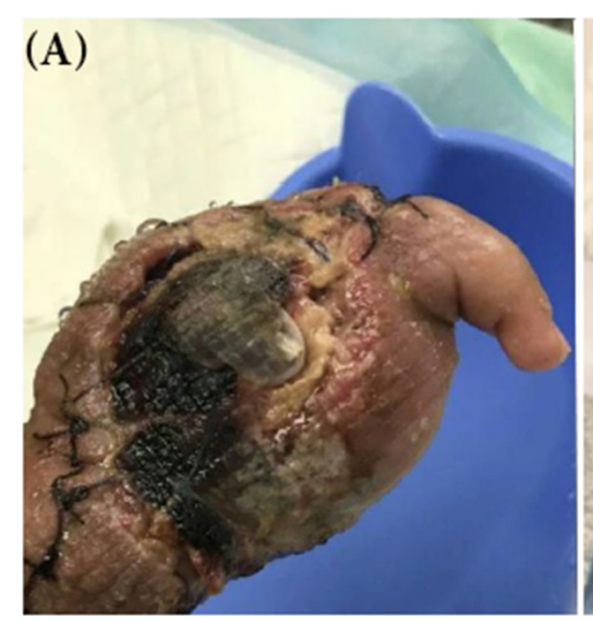

Figure 4. Necrosis and Amputation of the Fifth Finger

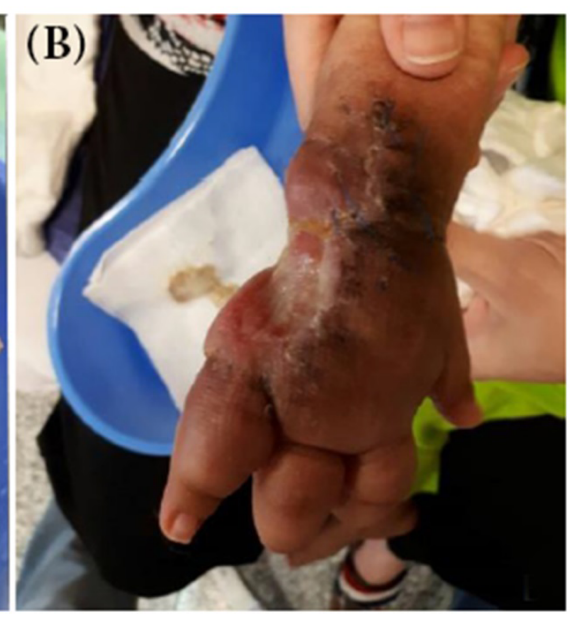

A: Necrosis of the fifth finger during the hospitalization period of the second surgery; B: Amputation of the fifth finger 

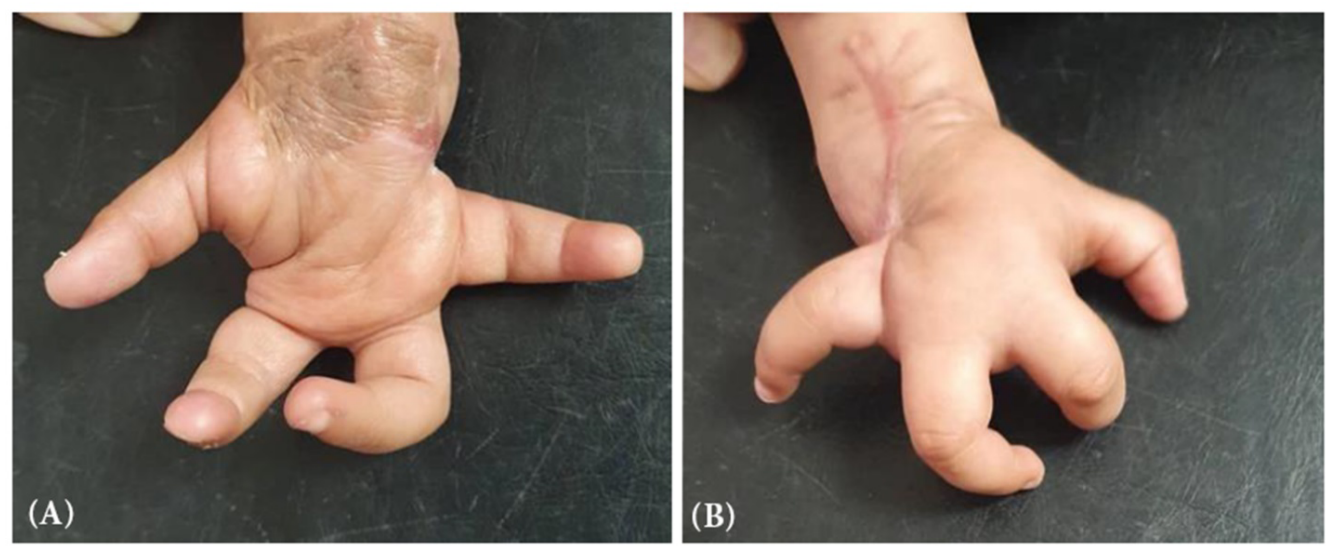

Figure 5. Photographs of the involved hand, one year after the second surgery

Orthoppedic Science

A: Volar side; B: Dorsal side

Hayek et al. reported two cases of infantile fibrosarcoma that were misdiagnosed as vascular tumors. The first case was a 2-month-old boy with a lesion on the ulnar side of the right hand. The lesion had a flat diameter of $10 \mathrm{~cm}$ and a bleeding ulcer on its apex. Ultrasound examination revealed several enlarged arterial and venous structures. Besides, the pathology report was peculiar. Finally, the immunohistochemical analysis confirmed the diagnosis of infantile fibrosarcoma. The second case was a $2 \times 2 \mathrm{~cm}$ mass with a bluish elevation of the skin. The mass was rapidly growing in size until it ulcerated and started to bleed. Although the lesion was initially diagnosed as a vascular mass, pathologic investigation suggested the diagnosis of infantile fibrosarcoma, which was confirmed by immunohistochemical analysis [7].

Hashemi et al. presented a case of a 9-year-old girl with a relapsing mass in her left hand. With a size of $3 \times 4 \mathrm{~cm}$, the mass had been initially noticed four years before the presentation and was gradually growing. The excised tumor had been pathologically diagnosed as a soft-tissue mass (lipoma). However, the tumor relapsed four years later, and invasive soft-tissue mass was suggested in MRI evaluation. The histologic reevaluation of the mass after relapse was consistent with the diagnosis of infantile fibrosarcoma. The authors pointed to the histological similarities to benign tumors as a cause of misdiagnosis [8].

$\mathrm{Hu}$ et al. reported a case of a 4-month-old girl who was presented with a congenital mass of right axillary. Clinically, the lesion mimicked a benign vascular/lymphatic malformation. However, immunohistochemical analysis was not consistent with a diagnosis of vascular lesions/ tumors. Finally, the molecular genetic analysis confirmed the diagnosis of infantile fibrosarcoma through the identification of positive ETS translocation variant 6/neurotrophic tyrosine kinase receptor, type 3 fusion transcript
[5]. This case suggests genetic analysis in the cases of infantile fibrosarcoma with peculiar patterns and challenging clinical, radiologic, and pathologic presentations.

Moreover, several investigations have reported the misdiagnosis of infantile fibrosarcoma with benign softtissue tumors [5]. These findings highlight the importance of considering infantile fibrosarcoma, when an infant presents with a lesion that clinically, radiologically, and even histologically mimics vascular lesion.

The diagnosis of infantile fibrosarcoma can be challenging as the lesion might clinically, radiologically, and even histologically mimics benign soft-tissue tumors. Therefore, further awareness is required to prevent the undertreatment of involved patients. Immunohistochemical and molecular genetic analyses are helpful in cases with a peculiar pattern.

\section{Ethical Considerations}

\section{Compliance with ethical guidelines}

The patient's parents provided written informed consent for publication of this report.

\section{Funding}

This research did not receive any specific grant from funding agencies in the public, commercial, or notprofit sectors.

\section{Authors' contributions}

Conceptualization: Sam Hajialiloo Sami, Farshad Zandrahimi; Data collection: Mohamadreza Heidarikhoo, Mahsa Zahmatkesh, Mansour Karimi; Drafting the manuscript: Hossein Gholampour. 


\section{Conflict of interest}

The authors declared no conflict of interest.

\section{References}

[1] Chung EB, Enzinger FM. Infantile fibrosarcoma. Cancer. 1976; 38(2):729-39. [DOI:10.1002/1097-0142(197608)38:2<729::aidcncr2820380216>3.0.co;2-z] [PMID]

[2] Ries LAG. Cancer incidence and survival among children and adolescents: United States SEER program, 1975-1995. Bethesda: National Cancer Institute; 1999. https://books. google.com/books/about/Cancer_Incidence_and_Survival_Among_Chil.html?id=LaxAFksCV6gC

[3] Pousti TJ, Upton J, Loh M, Grier H. Congenital fibrosarcoma of the upper extremity. Plast Reconstr Surg. 1998;102(4):115862. [DOI:10.1097/00006534-199809020-00037] [PMID]

[4] Parida L, Fernandez-Pineda I, Uffman JK, Davidoff AM, Krasin MJ, Pappo A, et al. Clinical management of infantile fibrosarcoma: A retrospective single-institution review. Pediatr Surg Int. 2013; 29(7):703-8. [DOI:10.1007/ s00383-0133326-4] [PMID] [PMCID]

[5] Hu Z, Chou PM, Jennings LJ, Arva NC. Infantile fibrosarcomaa clinical and histologic mimicker of vascular malformations: Case report and review of the literature. Pediatr Dev Pathol. 2013; 16(5):357-63. [DOI:10.2350/13-05-1335-CR.1] [PMID]

[6] Enos T, Hosler GA, Uddin N, Mir A. Congenital infantile fibrosarcoma mimicking a cutaneous vascular lesion: A case report and review of the literature. J Cutan Pathol. 2017; 44(2):193-200. [DOI:10.1111/cup.12854]

[7] Hayek SN, Janom HH, Ibrahim A, Moran SL. Infantile fibrosarcoma misdiagnosed as vascular tumors. Hand. 2013; 8(4):464-8. [DOI:10.1007/s11552-013-9519-4] [PMID] [PMCID]

[8] Hashemi A, Tefagh S, Seifadini A, Moghimi M. Infantile fibrosarcoma in a child: A case report. Iran J Ped Hematol Oncol. 2013; 3(3):135-7. [PMID] [PMCID] 
This Page Intentionally Left Blank 